\title{
Gasser M. Hathout, Tanya S. Ferguson: Clinical neuroradiology: a case-based approach
}

\author{
Cambridge University Press, 2008, (ISBN 9780521600545$) \$ 125 / £ 85$
}

\author{
David Summers
}

Received: 7 December 2009 /Accepted: 8 December 2009/Published online: 25 February 2010

(C) Springer-Verlag 2010

The authors' stated aim for this single-volume textbook is to bridge the gap between neurology and neuroradiology, providing imaging correlates for typical clinical neurology cases, and clinical correlates for neuroradiological appearances.

The book is divided into chapters by anatomical location, and each contains an anatomical description of the region, illustrated with line diagrams and pathological specimens or sections. This is followed by a series of clinical cases with clinical presentation, imaging findings, and discussion of the resulting appearances and relevant clinical notes. The imaging is of good quality throughout. There is a final chapter on imaging and therapy in stroke, which whilst slightly incongruous beside the anatomical approach that precedes it, does describe the recent history of stroke imaging including diffusion and perfusion imaging, and the evidence for thrombolysis and vascular intervention well.

The authors are clearly well-read and knowledgeable, and have an excellent collection of cases. The style of

D. Summers $(\bowtie)$

Department of Clinical Neurosciences, Western General Hospital, Edinburgh EH4 2XU, UK

e-mail: david.summers@ed.ac.uk presentation may, however, make extracting the information more difficult for some readers. The cases studies are text-rich, without many sub-headings, and are fairly discursive in style. The cases are often spread across pages requiring the reader to shuttle back and forth between images and discussion. Despite this, careful reading of the text does reveal a wealth of information and certainly extended my knowledge well beyond the neuroradiological necessities. Having the time to do this is the challenge in today's healthcare environment.

Knowing where to place this book in the current radiological literature is difficult. It is not a bench book, nor is it a beginner text, as much background knowledge is assumed. It would suit the inquisitive neuroradiologist wishing to expand his understanding from the imaging features into the anatomic-pathologic causes for the conditions described.

If you can find the time to do this book justice, and you can relate to the textual style which is quite different to most standard neuroradiology textbooks (you should probably try before you buy), then at $£ 85 / \$ 125$ this is a relatively inexpensive investment and a diverting readand you are likely to increase the breadth of your neurological knowledge considerably. 